\section{Géneros}

Multidisciplinary Journal of Gendet: Studies

\section{Hipatia Press}

www.hipatiapress.com

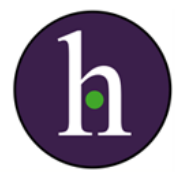

Instructions for authors, subscriptions and further details:

http://generos.hipatiapress.com

\title{
Critical Perspectives on Child Sexual Exploitation and Related Trafficking
}

Guiomar Merodio ${ }^{1}$

1) University of Barcelona. Spain

Date of publication: October $25^{\text {th }}, 2014$

Edition period: June 2014 - October 2014

To cite this article: Merodio, G. (2014). Critical Perspectives on Child Sexual Exploitation and Related Trafficking [Review of the book]. Multidisciplinary Journal of Gender Studies, 3(2), 556-558. doi: 10.4471/generos.2014.46

To link this article: http://dx.doi.org/10.447/generos.2014.46

\section{PLEASE SCROLL DOWN FOR ARTICLE}

The terms and conditions of use are related to the Open Journal System and to Creative Commons Attribution License (CC-BY). 
GÉNEROS -Multidisciplinary Journal of Gender Studies Vol. 3 No.3 June 2014 pp. 556-558

\section{Review}

Melrose, M. \& Pearce, J. (Ed). (2013). Critical Perspectives on Child Sexual Exploitation and Related Trafficking. Reino Unido: Palgrave Macmillian.

M

argaret Melrose y Jenny Pearce, destacadas investigadoras de la Universidad Bedfordshire de Reino Unido, abordan en este protección de menores víctimas de trata y/o explotación sexual. Mediante las contribuciones de otras investigadoras e investigadores, compilan información basada en evidencias sobre los diferentes escenarios en los que se producen este tipo de violencias, así como las innovaciones sociales que se están desarrollando en el Reino Unido.

En el primer capítulo Margaret Melrose realiza un análisis crítico del discurso dominante sobre explotación sexual de menores que fundamenta las políticas públicas del Reino Unido. Argumenta que la expansión reciente del concepto de explotación sexual ha provocado que éste se vuelva maleable y señala que este discurso está reproduciendo estereotipos sobre la infancia y situando a las víctimas afectadas en un rol pasivo, carentes de agencia.

Jon Pitts afronta en el segundo capítulo la problemática de la explotación sexual de menores en las pandillas juveniles. Mediante estudios empíricos, subraya la necesidad de superar las definiciones comunes de explotación sexual infantil que ignoran la complejidad de las situaciones de abuso y explotación. Así, revela datos alarmantes sobre el aumento de las violaciones en grupo perpetradas en Reino Unido contra mujeres y adolescentes, que han aumentado en un 150\% entre los años 2004 y 2008, disminuyendo cada vez más la edad de víctimas y perpetradores. 
Carlene Firmin, en el tercer capítulo, analiza si las conceptualizaciones actuales sobre abuso y violencia permiten identificar y responder eficazmente a las relaciones de abuso y violencia sexual que se producen en el grupo de iguales y defiende la necesidad de introducir la perspectiva de género en la definición y contextualización adecuada de este tipo de abuso.

El cuarto capítulo trata un tema fundamental para derribar falsas creencias y mitos en torno a las relaciones "consentidas" que generan una victimización secundaria por parte de instituciones y profesionales. Jenny Pearce considera cuatro tipologías explicativas para comprender cómo ese aparente consentimiento se encuentra coaccionado por diversas características y situaciones sociales límites que vulneran las capacidades y posibilidades de las personas.

Helen Beckett explora en el capítulo 5 las vulnerabilidades y deficiencias del sistema de protección de menores basándose en las conclusiones de un estudio realizado a gran escala en Irlanda del Norte, identificando, por otra parte, ejemplos de prácticas prometedoras.

En el siguiente capítulo, Isabelle Brodie, revisa estudios relacionados con la explotación sexual de menores en Escocia, analizando las diferentes políticas aplicadas para tratar el problema y si éstas se fundamentan en evidencias científicas. Más adelante, en el capítulo 9, Lucie Shuker aborda las conexiones entre los sistemas de protección y el riesgo de padecer abuso y explotación sexual. Además, expone un modelo para entender la importancia de atender tanto los aspectos físicos como psicológicos y relacionales en los procesos de recuperación de las víctimas, otorgando un papel importante a la comunidad.

Nicola Sharp resalta en el capítulo siete la importancia de incorporar una perspectiva interseccional para corregir el olvido de las mujeres y jóvenes negras y de minorías étnicas, y comprender que sus experiencias están mediatizadas por diferentes sistemas de desigualdad, con el fin de favorecer una adecuada valoración y protección de los riesgos de explotación sexual, como desapariciones, matrimonios forzados y violencia basada en el honor.

El capítulo ocho plantea un asunto relevante para la democratización de los procesos de intervención social: la necesidad de escuchar las voces de 
las víctimas de abusos y promover su participación activa en la toma de decisiones que les conciernen a su protección, como una manera de realizar una atención más adecuada y de promocionar su recuperación.

Por último, el libro señala contradicciones en las políticas públicas de la Unión Europea, indicando que existe una tendencia a individualizar el problema, encubriendo así las circunstancias sociales, económicas, políticas y culturales que sustentan globalmente las situaciones de explotación sexual y desigualdad.

En definitiva Critical perspectives on child sexual exploitation and related trafficking es una lectura académica recomendable para profesionales de la intervención social y para personal investigador interesado en contribuir a la superación radical de un problema de enormes proporciones que vulnera gravemente los derechos humanos.

Guiomar Merodio, University of Barcelona guiomar.merodio@gmail.com 